\title{
Mobility Effects of Wireless Multi-hop Networks in Indoor Scenarios
}

\author{
Makoto Ikeda*, Masahiro Hiyama*, Leonard Barolli ${ }^{\dagger}$, Fatos Xhafa ${ }^{\ddagger}$, Arjan Durresi ${ }^{\S}$ and Makoto Takizawa \\ ${ }^{*}$ Graduate School of Engineering, \\ Fukuoka Institute of Technology (FIT), \\ 3-30-1 Wajiro-Higashi, Higashi-Ku, Fukuoka 811-0295, Japan \\ Email: makoto.ikd@acm.org,mgm09007@bene.fit.ac.jp \\ ${ }^{\dagger}$ Department of Information and Communication Engineering, \\ Fukuoka Institute of Technology (FIT), \\ 3-30-1 Wajiro-Higashi, Higashi-Ku, Fukuoka 811-0295, Japan \\ Email: barolli@fit.ac.jp \\ ${ }^{\ddagger}$ Department of Languages and Informatics Systems, \\ Technical University of Catalonia, \\ Jordi Girona 1-3, 08034 Barcelona, Spain \\ Email: fatos@lsi.upc.edu \\ $\S$ Department of Computer and Information Science, \\ Indiana University Purdue University Indianapolis (IUPUI), \\ 723 W. Michigan Street SL 280M, Indianapolis, IN 46202, USA \\ Email:durresi@cs.iupui.edu \\ "Department of Computers and Information Science, \\ Seikei University, \\ 3-3-1 Kichijoji-kitamachi, Musashino-shi, Tokyo 180-8633, Japan \\ Email:makoto.takizawa@computer.org
}

\begin{abstract}
A Mobile Ad hoc Network (MANET) is a collection of wireless mobile terminals that are able to dynamically form a temporary network without any aid from fixed infrastructure or centralized administration. In recent years, MANETs are continuing to attract the attention for their potential use in several fields such as military activities, rescue operations and time-critical applications. In this paper, we present the implementation and analysis of our implemented wireless multi-hop network testbed considering the Optimized Link State Routing (OLSR) protocol for wireless multi-hop networking. We investigate the effect of mobility and topology changing in MANET. We study the impact of best-effort traffic for non line of sight communication. In this work, we consider three models: stationary, mobility and non line of sight communication models. We assess the performance of our testbed in terms of throughput and packet loss. From the experimental results, we found that OLSR has not a good performance when the relay node is moving. Also, the performance deteriorates when the CBR is higher.
\end{abstract}

Keywords-MANET; Testbed; Non Line of Sight; OLSR.

\section{INTRODUCTION}

A Mobile Ad hoc Network (MANET) is a collection of wireless mobile terminals that are able to dynamically form a temporary network without any aid from fixed infrastructure or centralized administration. The growth of laptops and wireless networking have made MANET a popular subject for research since the mid to late 1990s. In recent years, MANET are continuing to attract the attention for their potential use in several fields. Mobility and the absence of any fixed infrastructure make MANET very attractive for mobility and rescue operations and time-critical applications. A typical example of this approach has revealed many aspects of IEEE 802.11, like the gray-zones effect [1], which usually are not taken into account in standard simulators, as the well-known $n s-2$ simulator [2]. So far we can count a lot of computer simulation results on the performance of MANET, e.g. in terms of end-to-end throughput, delay and packet loss. However, in order to assess the computer simulation results, real-world experiments are needed and a lot of testbeds have been built to date [3]. The baseline criteria usually used in real-world experiments is guaranteeing the repeatability of tests, i.e. if the system does not change along the experiments. How to define a change in the system is not a trivial problem in MANET, especially if the nodes are mobile. 
In this paper, we concentrate on the performance analysis of a small testbed of one desktop and four laptops acting as nodes of a MANET. We use Optimized Link State Routing (OLSR) protocol, which is a pro-active routing protocol, and it has been gaining great attention within the scientific community. Furthermore, the olsrd [4] software we have used in our experiments is the most updated software we have encountered.

In our previous work, we found the following results. We proved that while some of the OLSR's problems can be solved, for instance the routing loop, this protocol still have the self-interference problem. There is an intricate interdependence between MAC layer and routing layer, which can lead the experimenter to misunderstand the results of the experiments. For example, the horizon is not caused only by IEEE 802.11 Distributed Coordination Function (DCF), but also by the routing protocol. We carried out the experiments considering stationary nodes of ad-hoc network. We considered the node mobility and carry out experiments for OLSR and B.A.T.M.A.N. protocols [5]. We found that throughput of TCP were improved by reducing Link Quality Window Size (LQWS), but there were packet loss because of experimental environment and traffic interference. For TCP data flow, we got better results when the LQWS value was 10. Moreover, we found that the node join and leave operations affect more the TCP throughput and RTT than UDP [6].

In this work, we investigate the effect of non line of sight communication of MANET. We implemented three MANET models and carried out experiments for different topologies.

The structure of the paper is as follows. In Section II, we present the related work. In Section III, we give a short description of OLSR. In Section IV, we present the testbed design and implementation. In Section V, we present experimental evaluation. Finally, conclusions are given in Section VI.

\section{RELATED WORK}

Many researchers performed valuable research in the area of wireless multi-hop network by real experiment or computer simulation [7], [8]. Most of them are focused on throughput improvement, but they do not consider mobility [9]. In [10], the authors implemented multi-hop mesh network called MIT Roofnet. Roofnet consists of about 50 nodes. They consider the impact of node density and connectivity in the network performance. The authors show that the multi-hop link is better than single hop link in terms of throughput and connectivity. In [11], the authors analyze the performance of an outdoor ad-hoc network, but their study is limited to reactive protocols such as Ad hoc On Demand Distance Vector (AODV) [12] and Dynamic Source Routing (DSR) [13]. The authors of [14] performs outdoor experiments of non standard pro-active protocols. Other adhoc experiments are limited to identify MAC problems, by providing insights on the one-hop MAC dynamics as shown in [15].

The closest work to ours is that in [16]. However, the authors did not care about the routing protocol. In [17], the disadvantage of using hysteresis routing metric is presented through simulation and indoor measurements. Our experiments are concerned with the interaction of transport protocols and routing protocol. Furthermore, we compare the performance of the testbed for three scenarios: stationary (STA), moving (MOVE) and Non Line of Sight (NLOS).

In [18], the authors presents an experimental comparison of OLSR using the standard hysteresis routing metric and the Expected Transmission Count (ETX) metric in a 7 by 7 grid of closely spaced Wi-Fi nodes to obtain more realistic results. The throughput results are similar to our previous work and are effected by hop distance [19].

\section{OLSR OVERVIEW}

The link state routing protocol that is most popular today in the open source world is OLSR from olsr.org. OLSR with Link Quality (LQ) extension and fisheye-algorithm works quite well. The OLSR protocol is a pro-active routing protocol, which builds up a route for data transmission by maintaining a routing table inside every node of the network. The routing table is computed upon the knowledge of topology information, which is exchanged by means of Topology Control (TC) packets. OLSR makes use of HELLO messages to find its one hop neighbors and its two hop neighbors through their responses. The sender can then select its Multi Point Relays (MPR) based on the one hop node which offer the best routes to the two hop nodes. By this way, the amount of control traffic can be reduced. Each node has also an MPR selector set which enumerates nodes that have selected it as an MPR node. OLSR uses TC messages along with MPR forwarding to disseminate neighbor information throughout the network. Host Network Address (HNA) messages are used by OLSR to disseminate network route advertisements in the same way TC messages advertise host routes.

OLSRv2 is currently being developed at IETF. It maintains many of the key features of the original protocol including MPR selection and dissemination. Key differences are the flexibility and modular design using shared components such as packet format packetbb and neighborhood discovery protocol.

In our OLSR code, a simple RFC-compliant heuristic is used [20] to compute the MPR nodes. Every node computes the path towards a destination by means of a simple shortestpath algorithm, with hop-count as target metric. In this way, a shortest path can result to be also not good, from the point of view of the packet error rate. Accordingly, recently olsrd has been equipped with the LQ extension, which is a shortest-path algorithm with the average of the packet error rate as metric. This metric is commonly called as the 


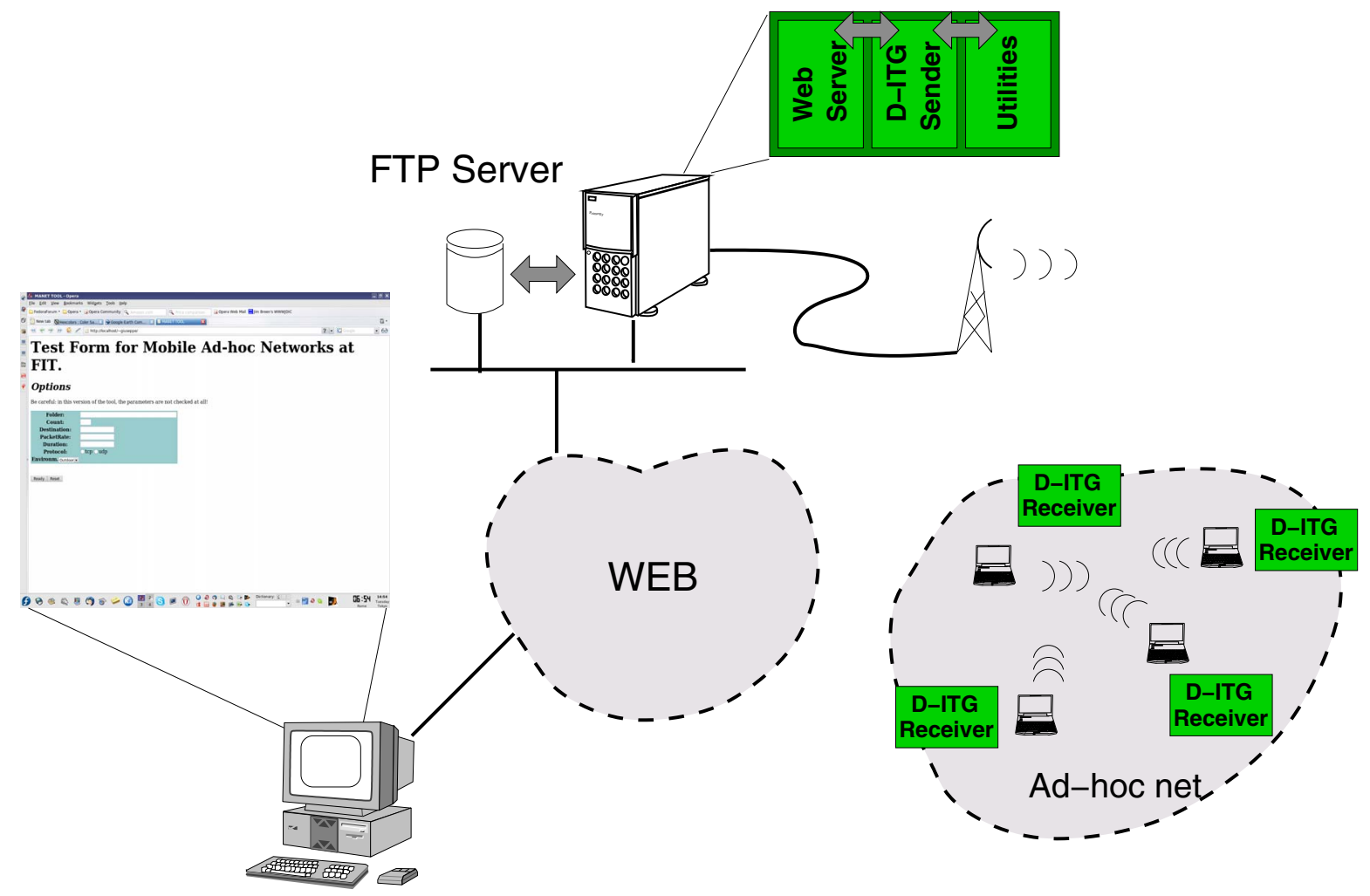

Figure 1. Testbed system overview.

ETX, which is defined as $\operatorname{ETX}(i)=1 /(N I(i) \times L Q I(i))$. Given a sampling window $W, \mathrm{NI}(i)$ is the packet arrival rate seen by a node on the $i$-th link during $W$. Similarly, LQI $(i)$ is the estimation of the packet arrival rate seen by the neighbor node which uses the $i$-th link. When the link has a low packet error rate, the ETX metric is higher. The LQ extension greatly enhances the packet delivery ratio with respect to the hysteresis-based technique [21].

\section{Testbed Design and Implementation}

\section{A. Target Environment}

We have implemented a MANET testbed which provides a realistic platform for analyzing various aspect of these networks, including the different topology models. For our testbed, we make the following considerations.

- We consider an indoor environment at our departmental floor.

- We investigate the effect of mobility and topology changing in the throughput of MANET testbed.

- We constructed three experimental models: Model 1 (all nodes are in stationary state); Model 2 (only one relay node is moving); Model 3 (four nodes are in stationary state and one relay node is leaving the network).

- The mobile nodes move toward the destination at a regular speed. When the mobile nodes arrive at the corner, they stop for about three seconds.

- In order to make the experiments easier, we implemented a testbed interface and web tool (see Fig. 1).

- Experimental time is 100 seconds.

\section{B. Testbed Description}

Our testbed is composed of four laptops and one gateway (GW) ${ }^{1}$ machine as shown in Figs. 2 - 4. We constructed three experimental models. The experimental parameters are shown in Table I. In Fig. 2, all nodes are in a stationary state. We call this model STA. The nodes position and movement are shown in Figs. 5(a), 5(b), 5(c), 5(e) and 5(f). In Fig. 3 , only one relay node (node id \#3) is moving. The mobile node moves toward the destination at a regular speed. When the mobile node arrives at the corner, it stops for about three seconds (see Fig. 5(d).). The round trip time is 100 seconds. We call this model MOVE. In the third model, one relay node id \#3 is leaving the network (see Fig. 4). We call this model NLOS.

The operating system mounted on these machines is Fedora Core 4 or Ubuntu 9.04 Linux with kernel 2.6.x, suitably modified in order to support the wireless cards. The wireless network cards are from Linksys. They are usb-based

\footnotetext{
${ }^{1} \mathrm{GW}$ node ID is \#1.
} 


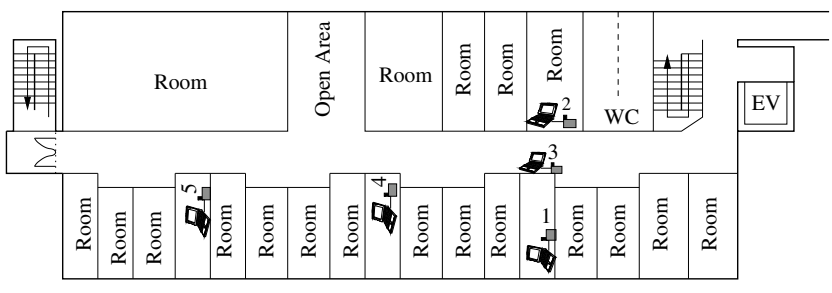

Figure 2. STA model (using iptables). Node \#1 is accessible via node \#3. When the destination node is \#2, the hop distance is 2 , i.e. $1 \rightarrow 3 \rightarrow 2$.

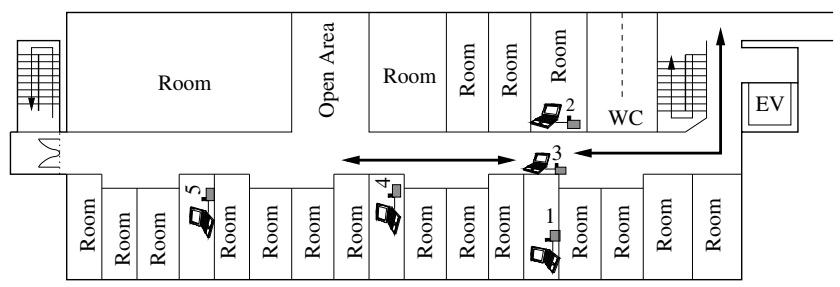

Figure 3. MOVE model (using iptables). The packet filtering rule is the same as STA.

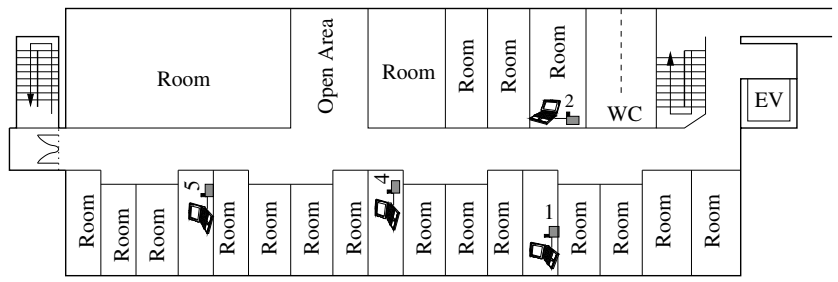

Figure 4. NLOS model (not using iptables). Between nodes there are some obstacles (metal doors and walls).

cards with and external antenna of $2 \mathrm{dBi}$ gain, transmitted power of $16+/-1 \mathrm{dBm}$ and receive sensitivity of $-80 \mathrm{dBm}$. We verified that the external antenna improves the quality of the first hop link, which is the link connecting the ad-hoc network. The driver can be downloaded from the web site in references [22], [23] ${ }^{2}$.

The source node serves as HTTP, FTP, DNS and Internet router for the nodes in the MANET. These features are provided by the iptables mechanism, readily available under Linux machines. The iptables is userspace command line program used to configure the Linux 2.4.x and 2.6.x IPv4 packet filtering ruleset. By this way, the GW can be accessed ubiquitously from anywhere. Moreover, the $\mathrm{GW}$ hosts also all the routines used to coordinate the measurement campaign, as well as graphical tools to check network connectivity. In our testbed, we have two systematic background or interference traffic we could not eliminate: the control traffic and the other wireless APs interspersed within the campus. The control traffic is due to the ssh program, which is used to remotely start and control the measurement software on the source node. The other traffic

\footnotetext{
${ }^{2}$ As far as we know the latest kernel include rt2500usb driver. However, this driver does not work for ad-hoc mode.
}

Table I

CLASSIFICATION OF NODES FOR EACH EXPERIMENTAL MODEL.

\begin{tabular}{c|c|c|c}
\hline Model & \multicolumn{2}{|c|}{ Number of moving nodes } & Number of stati- \\
\cline { 2 - 3 } & Source node & Relay node & onary nodes \\
\hline \hline STA & 0 & 0 & 5 \\
MOVE & 0 & 1 & 4 \\
NLOS & 0 & 0 & 4 \\
\hline
\end{tabular}

Table II

LIST OF THE EXPERIMENTAL PARAMETERS.

\begin{tabular}{c|c}
\hline Parameter & Value \\
\hline \hline Number of Nodes & 5 \\
MAC & IEEE 802.11 \\
Traffic Type & CBR \\
Packet Size & 512 bytes \\
Packet Rate & 122 or $244 \mathrm{pps}$ \\
Duration & $100000 \mathrm{msec}$ \\
Number of Trials & 10 \\
Protocol & OLSR \\
OLSR: Link Quality Window Size & 10 \\
\hline
\end{tabular}

is a kind of interference, which is typical in an academic scenario.

\section{Testbed Interface}

Until now, all the parameters settings and editing were done by using command lines of bash shell (terminal), which resulted in many misprints and the experiments were repeated many times. In order to make the experiments easier, we implemented a testbed interface. For the Graphical User Interface (GUI) we used wxWidgets tool and each operation is implemented by Perl language. wxWidgets is a cross-platform GUI and tools library for GTK, MS Windows and Mac OS X.

We implemented many parameters in the interface such as transmission duration, number of trials, source address, destination address, packet rate, packet size, LQWS, and topology setting function. We can save the data for these parameters in a text file and can manage in a better way the experimental conditions. Moreover, we implemented collection function of experimental data in order to make easier the experimenter's work.

\section{EXPERIMENTAL RESULTS}

\section{A. Experimental Settings}

The experimental parameters as shown in Table II. We study the impact of best-effort traffic for NLOS communication. In the STA and MOVE models, the MAC filtering routines are enabled. On the other hand, in the NLOS model, the MAC filtering routines are not enabled. We collected data for two metrics: the throughput and packet loss. These data are collected using the Distributed Internet Traffic Generator (D-ITG) [24], which is an open-source Internet traffic generator. 


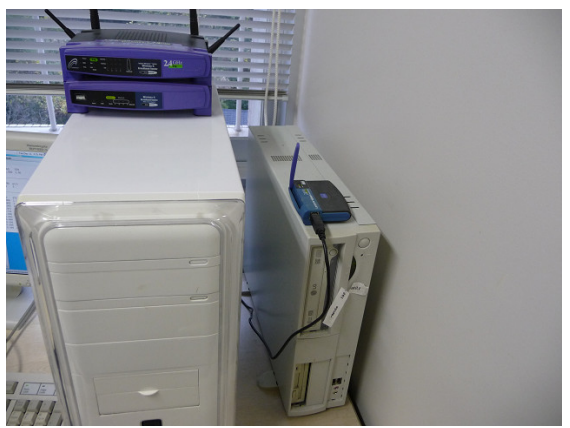

(a) Node ID \#1

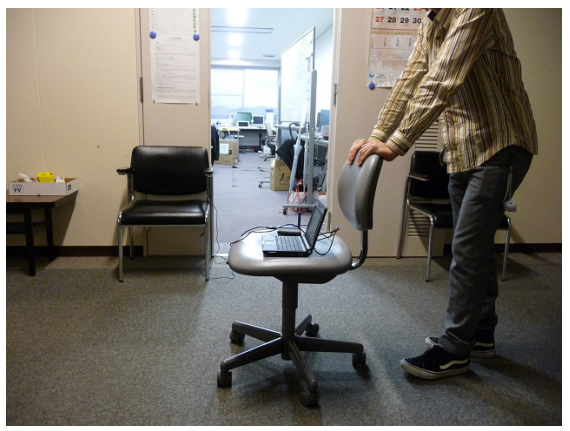

(d) Node ID \#3 (MOVE)

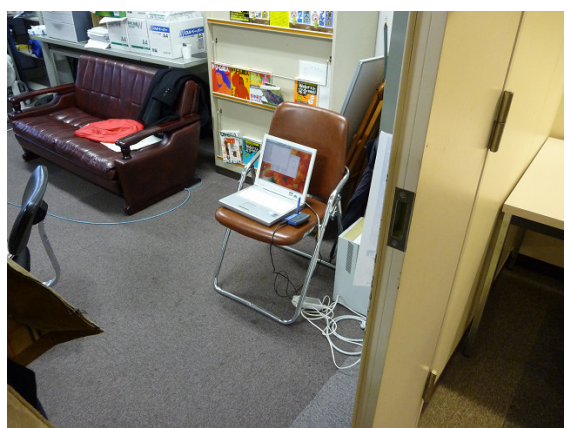

(b) Node ID \#2

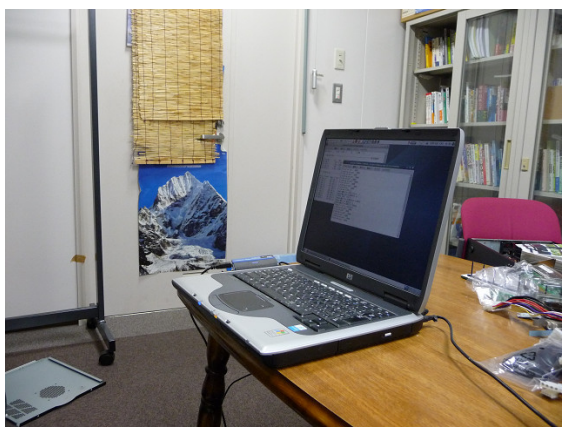

(e) Node ID \#4

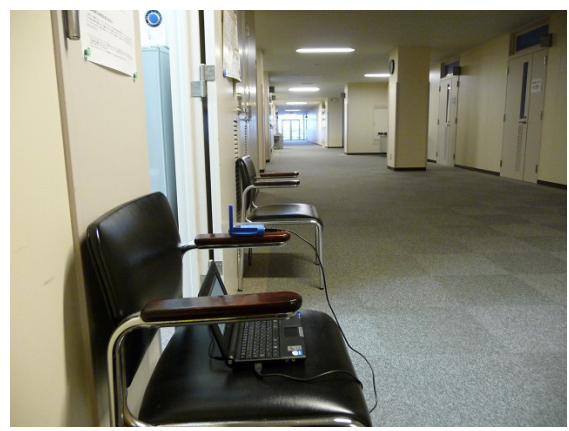

(c) Node ID \#3 (STA)

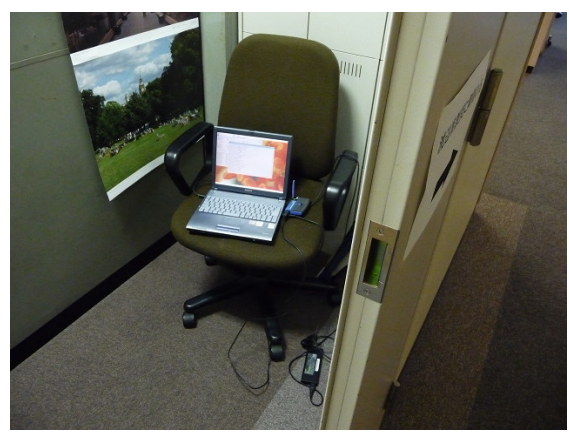

(f) Node ID \#5

Figure 5. Snapshot of each node.

In previous experiments [5], [19], [25], we realized that an external antenna improves radio signal reception. The Constant Bit Rate (CBR) of the data flows is 122 pps equal to $499.712 \mathrm{Kbps}$, i.e. the packet size of the payload is 512 bytes. All experiments have been performed in indoor environment, within our departmental floor of size roughly 100 meters. All nodes are in radio range of each other. In our previous work, one experiment lasted about 10 seconds and was repeated 50 times. But, the experimental time was very short. For this reason, in this paper we set the experimental time about 100 seconds. Moreover, we considered that the CBR of the data flows is 244 pps. We measured the throughput for UDP, which is computed at the receiver. We estimated the packet loss to compute the link quality metrics, e.g. LQ. For OLSR, $w T_{\text {HELLO }}<T_{\text {Exp }}$, where $T_{\operatorname{Exp}}$ is the total duration of the experiment, i.e., in our case, $T_{\mathrm{Exp}}=1000 \mathrm{~s}$, and $T_{\mathrm{HELLO}}$ is the rate of the HELLO messages. However, the testbed was turned on even in the absence of measurement traffic. Therefore, the effective $T_{\text {Exp }}$ was much greater.

As MAC protocol, we used IEEE 802.11. The transmission power was set in order to guarantee a coverage radius equal to the maximum allowed geographical distance in the network. Since we were interested mainly in the performance of the routing protocol, we kept unchanged all MAC parameters, such as the carrier sense, the retransmission counter, the contention window and the Request to Send (RTS) / Clear to Send (CTS) threshold. Moreover, the channel central frequency was set to $2.412 \mathrm{GHz}$ (channel 1). In regard to the interference, it is worth noting that, during our tests, almost all the IEEE 802.11 spectrum had been used by other access points disseminated within the campus. In general, the interference from other access points is a noncontrollable parameter.

\section{B. Experimental Measurements}

Results of our measurements are shown in Table III. All results showed the median values. In Table III, $1 \rightarrow 2$ means source node id $\rightarrow$ destination node id.

In order to show the range of variability of the data, we also report the box and whisker plot of the metrics according to the model types, as shown in Figs. 6 and 7. Box and whisker plot is a convenient way of graphically depicting groups of numerical data through their summaries: lower quartile (Q1), median (Q2), upper quartile (Q3), and the outliers. In the plots, the bottom and top of the box are always 25th and 75th percentile (Q1 and Q3, respectively), and the band near the middle of the box is always the median (Q2). The end of the whiskers can represent the lowest datum which is still within 1.5 inter-quartile range of the lower quartile, and the highest datum which is still within 1.5 inter-quartile range of the upper quartile.

In Figs. 6 and 7, the horizontal axis show the hop distance, i.e. $1 \rightarrow 2$ means source node id $\rightarrow$ destination node $i d$. In Fig. 6, the vertical axis shows the throughput (Kbps), which is computed at the receiver. In Fig. 7, the vertical axis shows 


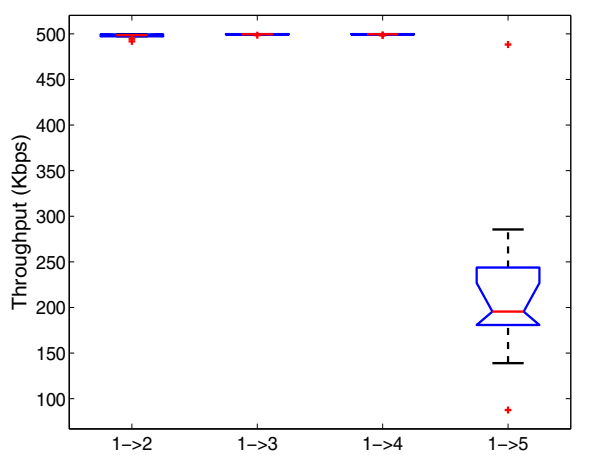

(a) $\mathrm{P}_{\text {Rate }}: 122$, STA

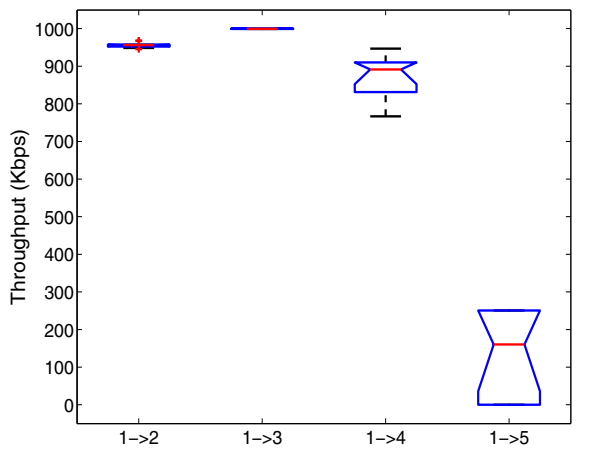

(d) $\mathrm{P}_{\text {Rate }}: 244, \mathrm{STA}$

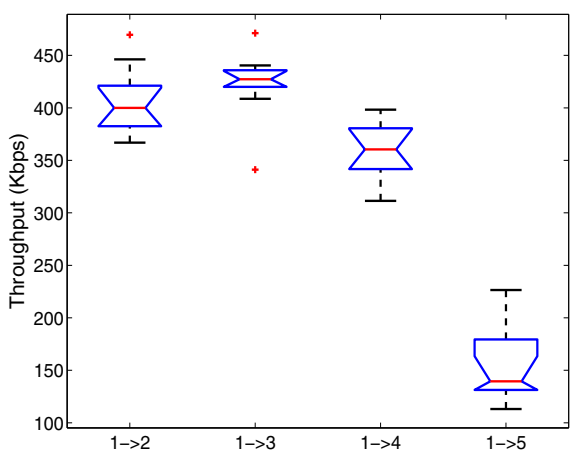

(b) $\mathrm{P}_{\text {Rate }}: 122, \mathrm{MOVE}$

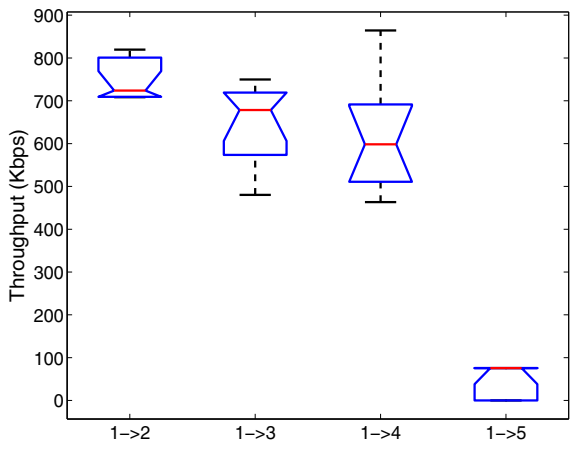

(e) $\mathrm{P}_{\text {Rate }}: 244$, MOVE

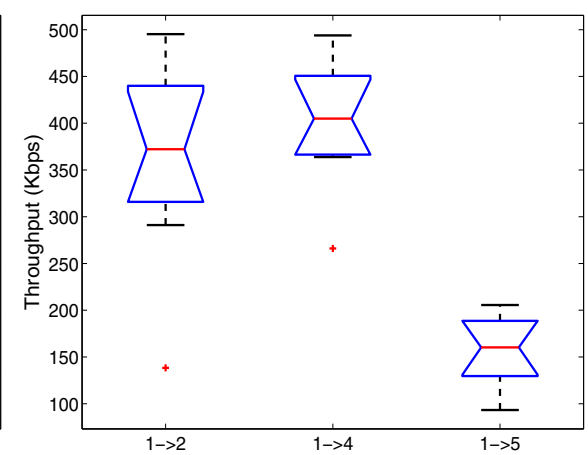

(c) $\mathrm{P}_{\text {Rate }}: 122$, NLOS

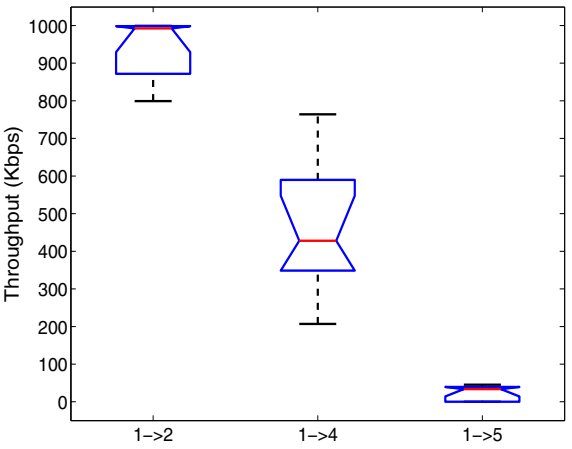

(f) $\mathrm{P}_{\text {Rate }}: 244, \mathrm{NLOS}$

Figure 6. Throughput results (Kbps).

Table III

COMPARISON MEDIAN THROUGHPUT (KBPS).

\begin{tabular}{cc|cccc}
\hline Model & $\mathrm{P}_{\text {Rate }}(\mathrm{pps})$ & $1 \rightarrow 2$ & $1 \rightarrow 3$ & $1 \rightarrow 4$ & $1 \rightarrow 5$ \\
\hline \hline STA & 122 & 498.6266 & 499.6096 & 499.6096 & 195.5271 \\
MOVE & 122 & 400.0288 & 427.3256 & 360.4263 & 139.5434 \\
NLOS & 122 & 372.1364 & - & 404.8876 & 160.2784 \\
\hline STA & 244 & 956.0780 & 999.3421 & 891.4298 & 160.4317 \\
MOVE & 244 & 723.9072 & 678.5305 & 598.2475 & 75.7077 \\
NLOS & 244 & 992.4608 & - & 427.9345 & 33.6073 \\
\hline \hline
\end{tabular}

the mean packet loss (pkt/sec), which is computed at the sender.

As you can see in Fig. 6(a) and (d), we can see a stable constant bit rate flow for each packet rate. But, we found that the throughput was decrease at $1 \rightarrow 5$. This is because of the hop distance. This fact show that the OLSR chooses correctly the 2 -hops or 3 -hops route, i.e. $1 \rightarrow 3 \rightarrow 5$ or $1 \rightarrow 3 \rightarrow 4 \rightarrow 5$.

In Fig. 6(b), (c), (e) and (f), after third hop, it seems that throughput is decreased. This is because of the effect of the relay node movement and it was caused by routing loops. Especially, when the CBR is 244 pps and the destination node is \#5, the throughput decreases significantly. During this experiment, we got a lot of error and can not communicate with node $\# 5$.

As shown in Fig. 7, there are some oscillations in each results. Moreover, the number of packet loss increases after node $1->4$ in Fig. 7. We can confirm these results also looking at Fig. 6. It seems that the topology can be very dynamic in MOVE. From these results, we see that the CBR data flow is affected more in MOVE compared with STA and NLOS. We found that OLSR protocol has not a good performance when the relay node is moving. Therefore, the OLSR protocol needs to be equipped with more realistic topology control mechanism in order to be used in different scenarios.

\section{CONCLUSIONS}

In this paper, we carried out experiments for a small MANET testbed with five nodes. We used OLSR protocol for real experimental evaluation.

In our experiments, we considered three models: STA, MOVE and NLOS. In STA, all nodes are in stationary state. In MOVE, one relay node is moving (every data flow have 


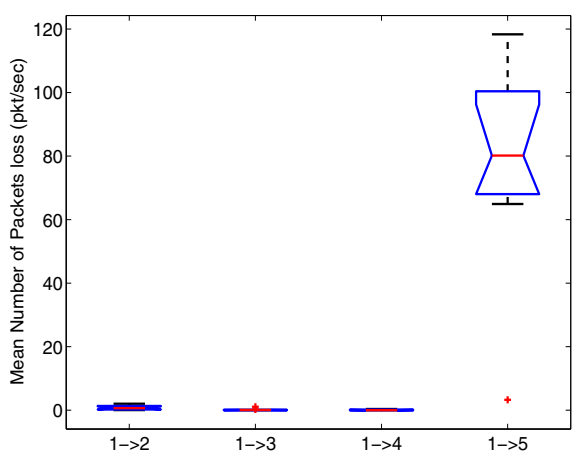

(a) STA, CBR:122

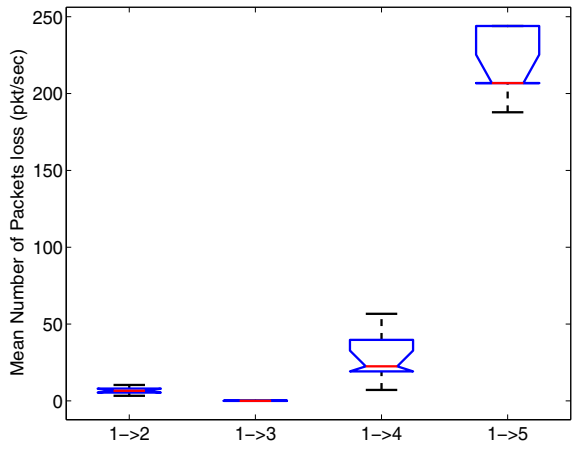

(d) STA, CBR:244

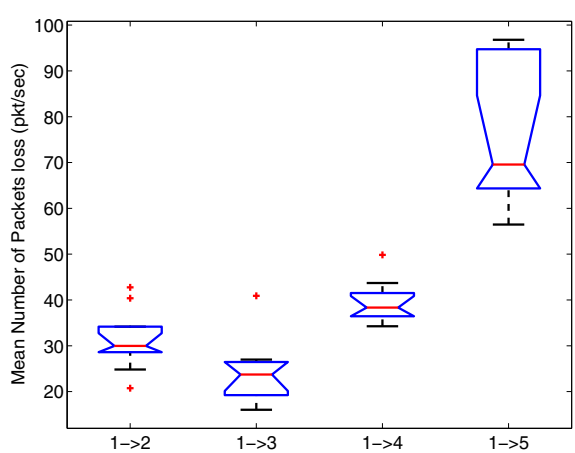

(b) MOVE, CBR:122

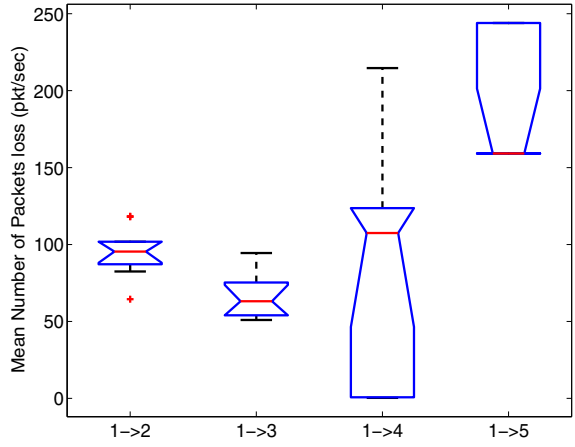

(e) MOVE, CBR:244

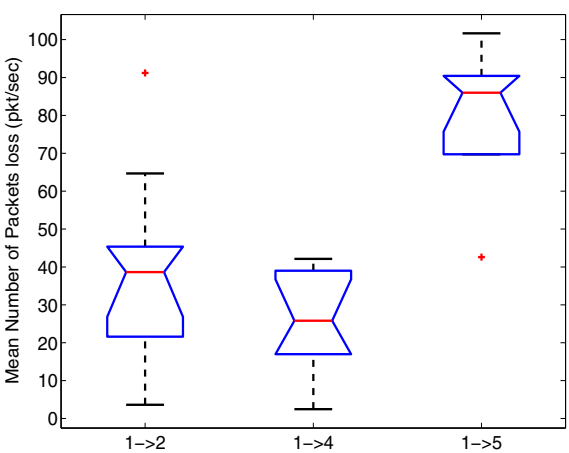

(c) NLOS, CBR:122

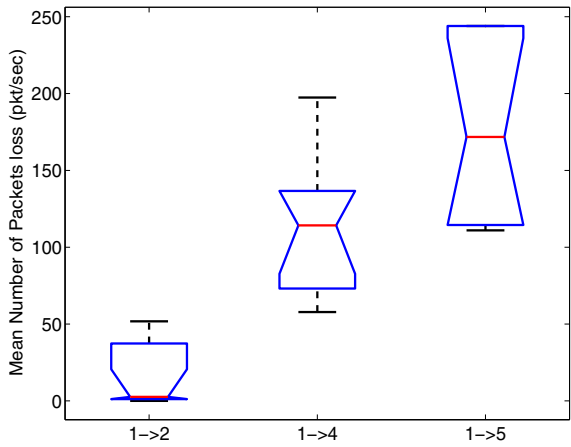

(f) NLOS, CBR:244

Figure 7. Packet loss results $(\mathrm{pkt} / \mathrm{sec})$.

Table IV

COMPARISON MEDIAN NUMBER OF PACKET LOSS (PKT/SEC).

\begin{tabular}{cc|cccc}
\hline Model & Packet rate $(\mathrm{pps})$ & $1 \rightarrow 2$ & $1 \rightarrow 3$ & $1 \rightarrow 4$ & $1 \rightarrow 5$ \\
\hline \hline STA & 122 & 0.6650 & 0.0600 & 0.0200 & 80.1690 \\
MOVE & 122 & 29.9692 & 23.7150 & 38.3418 & 69.5566 \\
NLOS & 122 & 38.6467 & - & 25.8031 & 85.9852 \\
\hline STA & 244 & 6.5962 & 0.0200 & 22.5031 & 206.8288 \\
MOVE & 244 & 95.4143 & 63.1298 & 107.4693 & 159.1765 \\
NLOS & 244 & 2.5995 & - & 114.2191 & 171.7375 \\
\hline \hline
\end{tabular}

to relay via this moving node). In NLOS, one relay node is leaving the network. We assessed the performance of our testbed in terms of throughput, and packet loss.

From our experiments, we found the following results.

- There are some oscillations in each model. This is because of hop distance and environment.

- The number of packet loss increases after 3 hop.

- We found that the OLSR protocol has not a good performance when the relay node is moving.

- The OLSR performance deteriorates when the CBR is higher.

These experiments where performed using OLSR routing protocol. In the future, we would like to consider the effect of reactive protocols and compare the experimental results with simulation results. Moreover, we would like to consider the new link quality metrics and extend our testbed.

\section{ACKNOWLEDGMENT}

This work is supported by a Grant-in-Aid for scientific research of Japan Society for the Promotion of Science (JSPS). The authors would like to thank JSPS for the financial support.

\section{REFERENCES}

[1] H. Lundgren, E. Nordström, and C. Tschudin, "Coping with communication gray zones in IEEE $802.11 \mathrm{~b}$ based ad hoc networks," in Proceedings of the 5th ACM International Workshop on Wireless Mobile Multimedia (WOWMOM), 2002, pp. 49-55.

[2] "The ns2 network simulator," http://www.isi.edu/nsnam/.

[3] W. Kiess and M. Mauve, "A survey on real-world implementations of mobile ad-hoc networks," Ad Hoc Networks, vol. 5, no. 3, pp. 324-339, 2007. 
[4] A. Tønnesen, "OLSRd: Implementation code of the OLSR," http://www.olsr.org/.

[5] L. Barolli, M. Ikeda, G. De Marco, A. Durresi, and X. Fatos, "Performance analysis of OLSR and BATMAN protocols considering link quality parameter," in Proc. AINA 2009, May 2009, pp. 307-314.

[6] M. Ikeda, L. Barolli, M. Hiyama, T. Yang, G. De Marco, and A. Durresi, "Performance evaluation of a manet testbed for differenet topologies," in The 12th International Conference on Network-Based Information Systems (NBiS-2009), Indianapolis, Auguest 2009, pp. 327-334.

[7] E. Nordström, "Ape - a large scale ad hoc network testbed for reproducible performance tests," Master's thesis, Uppsala University, 2002.

[8] Y. Owada, Y. Takahashi, T. Suda, H. Terui, F. Taki, T. Yagi, and K. Mase, "A large scale wireless mobile ad hoc network testbed," IEEE Vehicular Technology Conference, pp. 324328, September 2005.

[9] R. Draves, J. Padhye, and B. Zill, "Comparison of routing metrics for static multi-hop wireless networks," in SIGCOMM '04, 2004, pp. 133-144.

[10] J. Bicket, D. Aguayo, S. Biswas, and R. Morris, "Architecture and evaluation of an unplanned $802.11 \mathrm{~b}$ mesh network," in MOBICOM, 2005, pp. 31-42.

[11] D. A. Maltz, J. Broch, and D. B. Johnson, "Lessons from a full-scale multihop wireless ad hoc network testbed," IEEE Personal Communications, vol. 8, no. 1, pp. 8-15, February 2001.

[12] C. Perkins, E. Belding-Royer, and S. Das, "Ad hoc OnDemand Distance Vector (AODV) Routing," RFC 3561 (Experimental), Jul. 2003.

[13] D. B. Johnson, D. A. Maltz, and J. Broch, DSR: The Dynamic Source Routing Protocol for Multi-Hop Wireless Ad Hoc Networks, ser. Ad Hoc Networking. Addison-Wesley, 2001, ch. 5, pp. 412-420.

[14] R. S. Gray, D. Kotz, C. Newport, N. Dubrovsky, A. Fiske, J. Liu, C. Masone, S. McGrath, and Y. Yuan, "Outdoor experimental comparison of four ad hoc routing algorithms," in Proc. MSWiM '04, 2004, pp. 220-229.

[15] G. Anastasi, E. Borgia, M. Conti, and E. Gregori, "IEEE 802.11b ad hoc networks: Performance measurements," Cluster Computing, vol. 8, no. 2-3, pp. 135-145, 2005.

[16] V. Kawadia and P. R. Kumar, "Experimental investigations into tcp performance over wireless multihop networks," in Proc. of E-WIND-2005, 2005, pp. 29-34.

[17] T. Clausen, G. Hansen, L. Christensen, and G. Behrmann, "The optimized link state routing protocol, evaluation through experiments and simulation," in Proc. of IEEE Symposium on Wireless Personal Mobile Communications, September 2001, http://hipercom.inria.fr/olsr/wpmc01.ps.
[18] D. Johnson and G. Hancke, "Comparison of two routing metrics in OLSR on a grid based mesh network," Ad Hoc Networks, vol. 7, pp. 374 - 387, March 2009.

[19] G. De Marco, M. Ikeda, T. Yang, and L. Barolli, "Experimental performance evaluation of a pro-active ad-hoc routing protocol in out- and indoor scenarios," in Proc. AINA-2007, Canada, May 2007, pp. 7-14.

[20] T. Clausen and P. Jacquet, "Optimized link state routing protocol (OLSR)," RFC 3626 (Experimental), 2003.

[21] D. S. J. D. Couto, D. Aguayo, J. Bicket, and R. Morris, "A high-throughput path metric for multi-hop wireless routing," in Proc. MobiCom-2003, 2003, pp. 134-146.

[22] “Rt2x00 project," http://rt2x00.serialmonkey.com/.

[23] "Ralink RT2570 USB enhanced driver," http://homepages. tu-darmstadt.de/ p p_larbig/wlan/.

[24] A. Botta, A. Dainotti, and A. Pescape, "Multi-protocol and multi-platform traffic generation and measurement," INFOCOM 2007 DEMO Session, May 2007.

[25] M. Ikeda, L. Barolli, G. De Marco, T. Yang, and A. Durresi, "Experimental and simulation evaluation of OLSR protocol for mobile ad-hoc networks," in Proc. NBiS-2008, Turin, Italy, September 2008, pp. 111-121. 